\title{
Nonlinear Fourier Transform for Analysis of Coherent Structures in Dissipative Systems
}

\author{
I. S. Chekhovskoy, ${ }^{1,2}$ O. V. Shtyrina, ${ }^{1,2}$ M. P. Fedoruk, ${ }^{1,2}$ S. B. Medvedev, ${ }^{1,2}$ and S. K. Turitsyn ${ }^{1,3}$ \\ ${ }^{1}$ Novosibirsk State University, Novosibirsk 630090, Russia \\ ${ }^{2}$ Institute of Computational Technologies, SB RAS, Novosibirsk 630090, Russia \\ ${ }^{3}$ Aston Institute of Photonic Technologies, Aston University, Birmingham B4 7ET, United Kingdom
}

(Received 16 November 2018; published 15 April 2019)

\begin{abstract}
Using the cubic Ginzburg-Landau equation as an example, we demonstrate how the inverse scattering transform can be applied to characterize coherent structures in dissipative nonlinear systems. Using this approach one can reduce the number of the effective degrees of freedom in the system when the dynamic is dominated by the coherent structures, even if they are embedded in the dispersive waves and demonstrate unstable behavior.
\end{abstract}

DOI: 10.1103/PhysRevLett.122.153901

The conventional Fourier transform is one the most holistic mathematical methods widely used in science and engineering. The Fourier transform has two key important functions that make it a universal engineering tool. First, it can make it possible to present objects under study in a simpler form, for example, an irregular temporal waveform as a set of several spectral harmonics. Second, it might facilitate analysis, for example, in some linear equations, where spectral harmonics evolve independent of each other and in a simple manner, enabling the straightforward description of otherwise complex dynamics in the Fourier domain.

Something similar is available for certain classes of the nonlinear equations integrable by the inverse scattering transform (IST) [1-6], also known as the nonlinear Fourier transform (NFT). In particular, the nonlinear Schrödinger equation (NLSE) is a well known and practically important example of such integrable equations [2]:

$$
i \frac{\partial U}{\partial z}+\frac{1}{2} \frac{\partial^{2} U}{\partial t^{2}}+|U|^{2} U=0
$$

Here, we consider only the focusing case (anomalous dispersion in the context of fiber-optic applications) and solutions $U(z, t)$ decaying at $t \rightarrow \pm \infty$ for all $z$. The NLS equation has a remarkable property that links its solutions to the spectrum of a linear operator: the Zakharov-Shabat spectral problem. The Zakharov-Shabat problem (ZSP) for potential $U(z, t)$ and a spectral parameter $\zeta=\xi+i \eta$ is written as

$\frac{\partial f}{\partial t}=-i \zeta f+U(z, t) g, \quad \frac{\partial g}{\partial t}=-U^{*}(z, t) f+i \zeta g$

The spectral parameter $\zeta$ defines here a continuous or discrete spectrum associated with the field $U(z, t)$. All details can be found in Refs. [2-6], so we only review the key points briefly. Without loss of generality, we will use optical terminology typical for signal propagation in optical fibers. The complete NFT spectrum of the considered ZSP includes (i) a continuous spectrum that is defined on the real axis of the complex plane $\zeta=\xi$ by the complex function $r(\xi)$, and (ii) a discrete spectrum that is given by $4 \times N$ real parameters (the set of complex-valued eigenvalues $\left\{\zeta_{n}\right\}$ having a positive imaginary part together with complex-valued norming constants $\left\{r_{n}\right\}$ ). The discrete eigenvalues correspond to a soliton part of the field distribution, with $N$ being the total number of solitons in the propagating signal $U(z, t)$. The discrete nonlinear spectrum defines the soliton content of an arbitrary propagating waveform $U(z, t)$. For the field $U(z, t)$ that consists of a set of separated solitons, each eigenvalue $\zeta_{n}$ specifies the soliton parameters: amplitude $2 \operatorname{Im}\left(\zeta_{n}\right)$, frequency $-2 \operatorname{Re}\left(\zeta_{n}\right)$, position $T_{n}=\log \left[\left|r_{n}\right| /\left(2 \operatorname{Im} \zeta_{n}\right)\right] /\left(2 \operatorname{Im} \zeta_{n}\right)$, and phase $\varphi_{n}=-\arg \left(i r_{n}\right)$.

For the NLSE, the field energy can be presented as a sum of continuous (dispersive waves) and discrete (solitons) spectra:

$$
\int_{-\infty}^{\infty}|U(z, t)|^{2} d t=\sum_{n=1}^{N} 4 \eta_{n}-\frac{1}{\pi} \int_{-\infty}^{\infty} \log |a(\xi)|^{2} d \xi,
$$

where the left side of equality corresponds to the energy calculated in the temporal domain $E_{t}(z)$, while the right side consists of a contribution of the discrete spectrum energy $E_{d}(z)$ and the continuous spectrum $E_{c}(z)$, so $E_{t}=E_{c}+E_{d}$. Application of NFT for the integrable NLSE has been well studied and documented [2-6]. Here, we explore the potential of its application in the dissipative, nonintegrable systems for characterization of coherent structures. There are, of course, many other wellstudied transforms in mathematics. The key question is why one would apply this particular transform and what 
one would get from this? We would like to stress again that IST/NFT cannot be applied in the same way in the considered dissipative system, as it is used in the integrable NLSE. However, in this Letter, we demonstrate that by applying IST/NFT to a dissipative system (then it is not supposed to work), one can observe that just a few discrete eigenvalues can present well the evolution of dissipative coherent structures. The proposed approach does not allow us to fully describe dissipative nonlinear system evolution, but it characterizes dynamics with good accuracy in situations when a discrete spectrum component (in the sense of the ZS spectral problem) is dominant.

We consider a particular albeit important example: the evolution of the field governed by the cubic GinzburgLandau Eq. (CGLE) in the context of laser system modeling (see, e.g., Ref. [7]):

$i \frac{\partial U}{\partial z}+\frac{1}{2} \frac{\partial^{2} U}{\partial t^{2}}+|U|^{2} U-i\left(\sigma U+\alpha \frac{\partial^{2} U}{\partial t^{2}}+\delta|U|^{2} U\right)=0$,

where $\delta>0$ is typically related to the effective distributed saturable absorber action and $\alpha>0$ describes the effect of distributed optical filtering. The saturated gain $g_{0}$ and linear loss $\Gamma$ define $\sigma$ :

$\sigma(E)=\frac{g_{0}}{2\left(1+E / E_{\mathrm{sat}}\right)}-\frac{\Gamma}{2}, \quad E(z)=\int|U(z, t)|^{2} d t$.

The CGL equation has special solutions in the form of chirped dissipative solitons:

$U(z, t)=U_{0}^{1+i C}(t) \exp \{i \phi z\}, \quad U_{0}(t)=\frac{A}{\cosh (t / \tau)}$.

The CGL Eq. (4) is not integrable; however, we can still formally compute the NFT (nonlinear) spectrum of the optical field $U(z, t)$ at each point $z$ and study an evolution of the nonlinear spectrum with $z$ (see also Ref. [8]). We anticipate that NFT might allow us to describe the evolution of coherent structures with a smaller number of parameters compared to conventional Fourier harmonics analysis. The Zakharov-Shabat problem (2) has been solved numerically using the Bofetta-Osborne method [9]. A combined method was used to localize discrete eigenvalues, including the preliminary finding of the zeros of the function $a(\zeta)$ using contour integrals, and their subsequent refinement using the Newton method [10].

To explain the approach, let us first apply NFT analysis to the general chirped pulse given by Eq. (6) (with independent $A$ and $C$ ). Figure 1 shows a number of discrete eigenvalues $N$ as a function of two chirped soliton parameters: the chirp $C$ and a product of the amplitude $A$ and the width $\tau$. The $A \tau$ parameter is proportional to the $L_{1}$ norm and also corresponds to the square root of the ratio

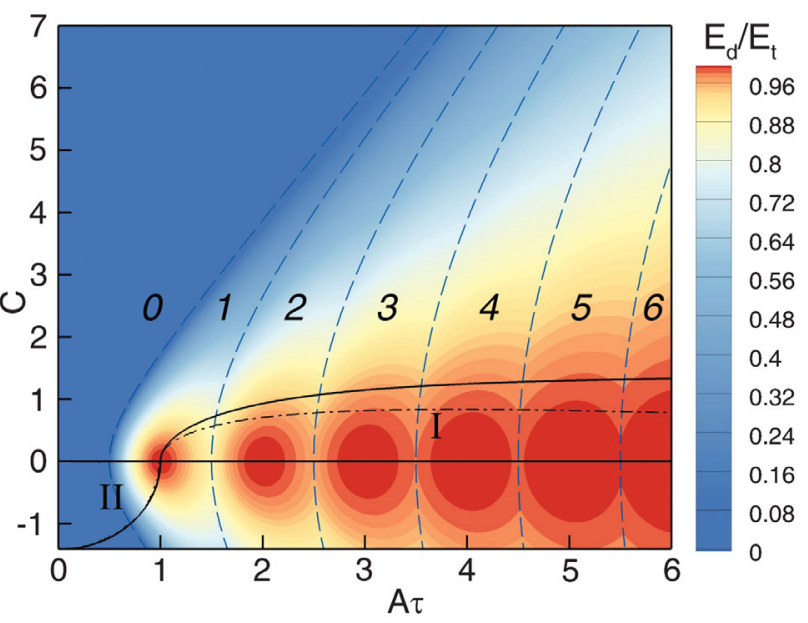

FIG. 1. Dependence of the ratio of the energy $E_{d}$, corresponding to the discrete spectrum, to the total energy $E_{t}$ on the $A \tau$ and the chirp parameter $C$ of the general potential given by Eq. (6). The numbers on the plot denote the number of discrete eigenvalues in the corresponding areas. Blue dashed lines defined by the analytical expression $A^{2} \tau^{2}=C^{2} / 4+(N-1 / 2)^{2}$ separate areas with different numbers of discrete eigenvalues. Areas limited by solid lines (I and II) define the subset of the CGLE solitons with parameters defined by coefficients in Eq. (4). The dash-dotted line denotes an existence domain of the chirped CGLE solitons for $g_{0}=0.3, \Gamma=0.1, E_{\mathrm{sat}}=1$.

$L_{D} / L_{\mathrm{NL}}$ of the effective dispersion length $\left(L_{D}=\tau^{2}\right)$ to the characteristic nonlinear length $\left(L_{\mathrm{NL}}=1 / A^{2}\right)$. The numbers on the plot denote areas with different numbers of discrete eigenvalues. A direct numerical solution of the ZakharovShabat spectral problem is compared with the analytical solution (which can be easily derived from the results of Refs. [11-15]): $A^{2} \tau^{2}=C^{2} / 4+(N-1 / 2)^{2}$ demonstrating perfect agreement. The set of eigenvalues $\left\{\zeta_{n}\right\}$ is defined as $\zeta_{n}=i \eta_{n}=i\left(\sqrt{A^{2} \tau^{2}-C^{2} / 4}+1 / 2-n\right)$, where $n$ are positive integers satisfying condition $\sqrt{A^{2} \tau^{2}-C^{2} / 4}+1 / 2-n>0$, providing for $\operatorname{Im}\left(\zeta_{n}\right)>0$. The colors in Fig. 1 display a fraction of the energy $E_{d}$, corresponding to the discrete spectrum, to the total energy $E_{t}$. We derive the analytical expression for the fraction of energy containing in the discrete spectrum,

$$
\frac{E_{d}}{E_{t}}=\frac{A^{2} \tau^{2}-C^{2} / 4-(\rho-1 / 2)^{2}}{A^{2} \tau^{2}},
$$

where $\rho=\operatorname{frac}\left[\sqrt{A^{2} \tau^{2}-C^{2} / 4}+1 / 2\right]$ is a fractional part of $\sqrt{A^{2} \tau^{2}-C^{2} / 4}+1 / 2$.

Next we examine the NFT spectra of the chirped solitons (6) that are specific solutions of the CGL equation. In this case, parameters $A, C$, and $\tau$ are not independent, but defined by $\delta, \alpha, g_{0}, \Gamma$, and $E_{\text {sat }}$. For $\delta>0$ and $\alpha>0$ chirp parameter $C$ is found as 


$$
C=-\frac{3}{2} \frac{(\delta \alpha+1 / 2)}{(-\delta / 2+\alpha)} \pm \frac{1}{2} \sqrt{9 \frac{(\delta \alpha+1 / 2)^{2}}{(-\delta / 2+\alpha)^{2}}+8}
$$

Here the sign in the above equation is determined from the condition $C /(-\delta / 2+\alpha)>0$. It is convenient to denote $R(C, \alpha)=\alpha\left(C^{2}-1\right)+C$ and $B(C, \alpha)=3 \alpha C+1-C^{2} / 2$, then soliton amplitude $A$, pulse width parameter $\tau$ and phase $\phi$ are given by $A^{2}=\sigma B(C, \alpha) / R(C, \alpha), \tau=$ $\sqrt{R(C, \alpha) / \sigma} \quad$ and $\quad \phi=\sigma\left[2 \alpha C-\left(C^{2}-1\right) / 2\right] / R(C, \alpha)$, respectively.

The value of steady-state energy $E_{s}$ is expressed either as $E_{s}=2 A^{2} \tau=2 B(C, \alpha) \sqrt{\sigma / R(C, \alpha)}$ or as $E_{s}=$ $E_{\text {sat }}\left[g_{0} /(2 \sigma+\Gamma)-1\right]$, see Eq. (5). This yields the equation on the energy $E_{s}$ of stationary solutions:

$$
\frac{R(C, \alpha)}{2 B^{2}(C, \alpha)} E_{s}^{2}=\frac{g_{0}}{\left(1+E_{s} / E_{\mathrm{sat}}\right)}-\Gamma .
$$

After energy $E_{s}$ is found, it fully determines parameter $\sigma$, the sign of which will be important in the following consideration.

The existence domain of the CGLE chirped solitons is determined only by the choice of the gain $g_{0}, \Gamma$, and $E_{\text {sat }}$ parameters and it can be asymptotically expanded to the quadrant $\delta \geq 0, \quad \alpha \geq 0 \quad$ with $\quad E_{\text {sat }} \times\left(g_{0}-\Gamma\right) / \Gamma \rightarrow 0$. Figure 2 illustrates this statement. Numerical modeling was carried out for the parameters $g_{0}=0.3, \Gamma=0.1$, $E_{\text {sat }}=1$ (which gives the energy value $E_{s}=E_{\text {sat }} \times\left(g_{0}-\right.$ $\Gamma) / \Gamma=2$ at $\delta=\alpha=0$ ), as well as for the parameters $g_{0}=0.6, \quad \Gamma=0.4, \quad E_{\text {sat }}=1 \quad\left[E_{\text {sat }} \times\left(g_{0}-\Gamma\right) / \Gamma=1 / 2\right]$. First, we compute the dependence of the characteristics of the nonlinear spectrum of the CGLE stationary solutions (4) on the parameters $\alpha$ and $\delta$, with all others fixed as described above. Figure 2 presents the ratio of the energy of the discrete part of the nonlinear spectrum $E_{d}$, to the total energy $E_{t}$ as a function of the parameters $\alpha$ and $\delta$. To
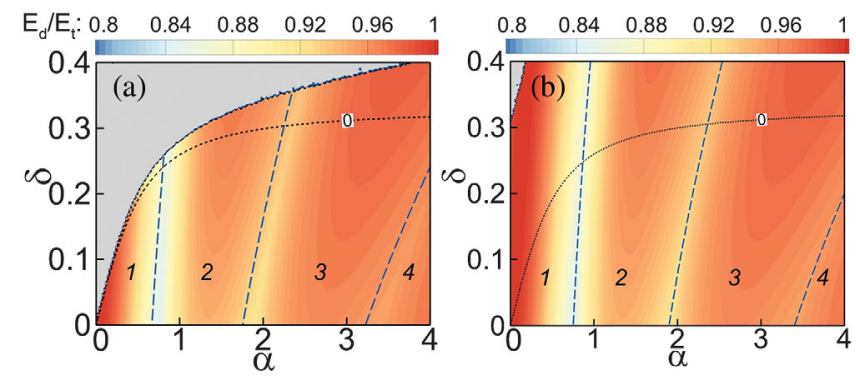

FIG. 2. The ratio of the discrete spectrum energy $E_{d}$ to the total energy $E_{t}$ of the steady state chirped soliton solution of the CGLE Eqs. (4)-(7) is shown as a function of the parameters $\alpha$ and $\delta$. Here $g_{0}=0.3, \Gamma=0.1$ (a) and $g_{0}=0.6, \Gamma=0.4$ (b). The dashed lines separate the areas with different eigenvalue counts for the corresponding CGLE stationary solutions Eqs. (6) and (7). The dotted line shows the level of $\sigma=0$. construct this figure, 200 values of each of the parameters $\alpha$ and $\delta$ were used. For each pair of parameters, continuous and discrete ZSP spectra have been numerically found for the corresponding stationary solution of the CGLE Eqs (6)-(7). The number of discrete eigenvalues for each set of parameters is plotted in the corresponding areas in Fig. 2(a). One can see that the proportion of the energy contained in the discrete spectrum to the total energy is always quite high-more than $82 \%$. However, the areas with 1 and 3 discrete eigenvalues have subdomains, where the energy ratio exceeds $97.5 \%$. This indicates that dynamics is dominated by the coherent structures and NFT might be the appropriate way to reduce an effective number of the degrees of freedom required for description of this system. The high concentration of energy in the discrete spectrum might be explained by the fact that the chirp value in the considered soliton solutions of the CGLE is limited: $|C|<\sqrt{2}$. Note that the purely discrete nonlinear spectrum is possible only when $C=0$ (when $2 \alpha=\delta$ ). In this case, $A \tau=1$. Figure 1 shows the complete existence domain of the chirped CGLE solitons. Only when $C=0$, the total energy is defined by the discrete one $E_{d} / E_{t}=1$ that corresponds to the purely discrete nonlinear spectrum. This indicates that NFT analysis might be useful for description of soliton dynamics in the CGLE. Our NFT based analysis, in particular, fully determine the dynamics of chirped pulses having the form Eq. (6) when they are launched into an optical fiber span with negligible loss effects (e.g., short nonlinear distance) $L_{\mathrm{NL}}$.

The boundaries separating areas with different discrete eigenvalue count (from 1 to 4 ) obtained numerically are in full agreement with the analytical formulas given the numbers of discrete eigenvalues $N$.

We now examine application of the NFT for analysis of CGLE Eq. (4) dynamics. A numerical modeling was performed using the standard split-step Fourier method. We consider as the initial condition the NLSE soliton $U(z=0, t)=0.2 / \cosh (0.2 t)$, with the peak power, that is less than the power of the stationary solutions of the CGLE Eq. (6) with the given parameters $\alpha$ and $\delta$.

Thus, the initial field always has only a single discrete eigenvalue, and the peak power of this field increases with propagation along $z$. We consider two situations: one with unstable dynamics with $\sigma>0$, and one with stable propagation with $\sigma<0$. For each of these examples, the nonlinear spectrum was calculated at 400 points along $z$. Figure 3 corresponds to the unstable case $(\sigma>0)$. Oscillations of the field intensity distribution are accompanied by the spatial variations of the discrete spectrum. It can be seen that, from the initial one discrete eigenvalue, the discrete spectrum is growing into several eigenvalues that contain most of the field energy. Two eigenvalues evolve stably with $z$. Two others appear and decay periodically with propagation. The periodically arising fourth discrete eigenvalue merges with the closest discrete 


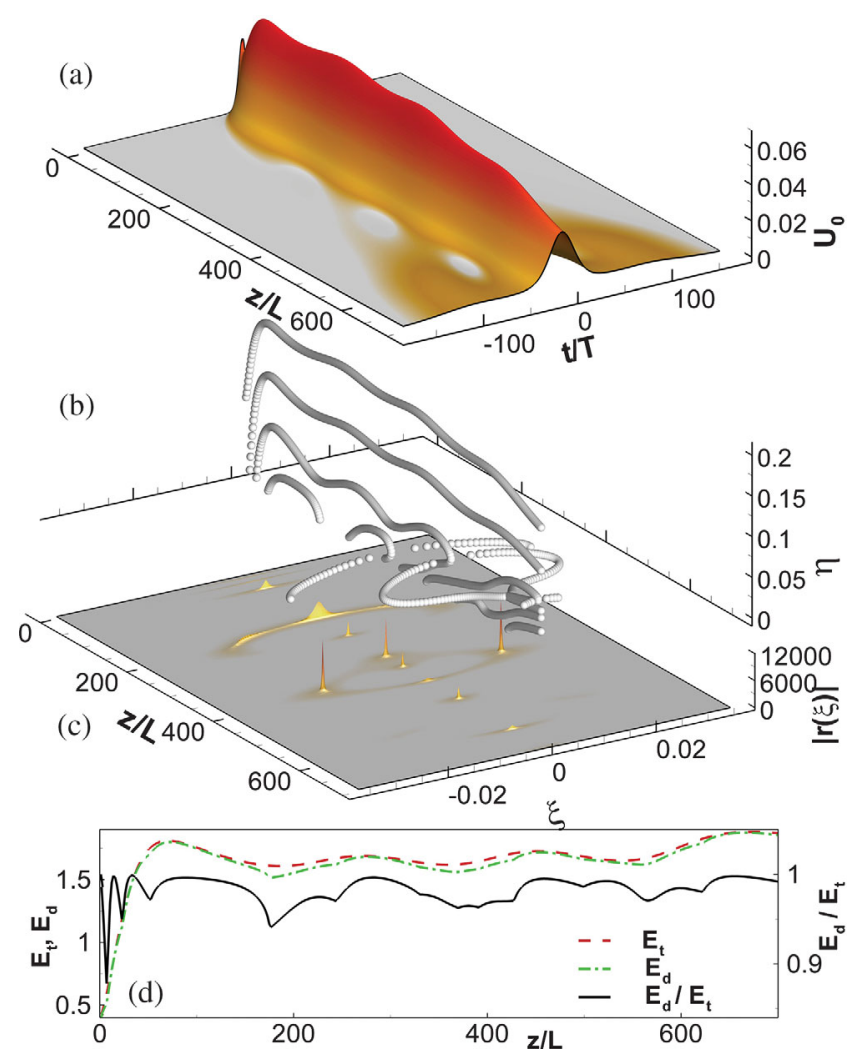

FIG. 3. (a) Evolution of the intensity of the initial pulse having form of the NLSE soliton. Here, $\alpha=3.5, \delta=0.15, \sigma>0$. (b) Dynamics of the discrete eigenvalues $\left\{\zeta_{n}\right\}$ of the nonlinear spectrum for the considered case and (c) the module of the continuous nonlinear spectrum $r(\xi)$. (d) Evolution of the total energy $E_{t}$, the discrete spectrum energy $E_{d}$, and the ratio $E_{d} / E_{t}$.

point in the NFT spectrum, leading to quasisymmetric configuration of these two eigenvalues relative to the real axis and out-of phase oscillations. Note that the continuous spectrum has fingerprints of the pulse features [see Fig. 3(c)]. The important observation depicted in Fig. 3(c) is that the major part of the energy is in the discrete spectrum, making NFT an attractive approach to reduce the number of degrees of freedom, even analyzing such unstable dynamics. Figure 4 shows the dynamics along $z$ of the field intensity and the dynamics of discrete eigenvalues $\zeta_{n}=\xi_{n}+\eta_{n}$ in the case of $\alpha=3.5, \delta=0.35$, $\sigma<0$. The initial single discrete eigenvalue evolves into three clearly seen discrete eigenvalues that correspond to the discrete NFT spectrum of the chirped CGLE soliton (6) for the considered values of the parameters. Oscillations that appear at first quickly fade out, and the fourth discrete eigenvalue that appeared at the beginning disappears. Again the lion's fraction of the energy is in these three discrete eigenvalues.

Using the Ginzburg-Landau equation as a model example, we applied the nonlinear Fourier transform method to characterize localized coherent structures in dissipative

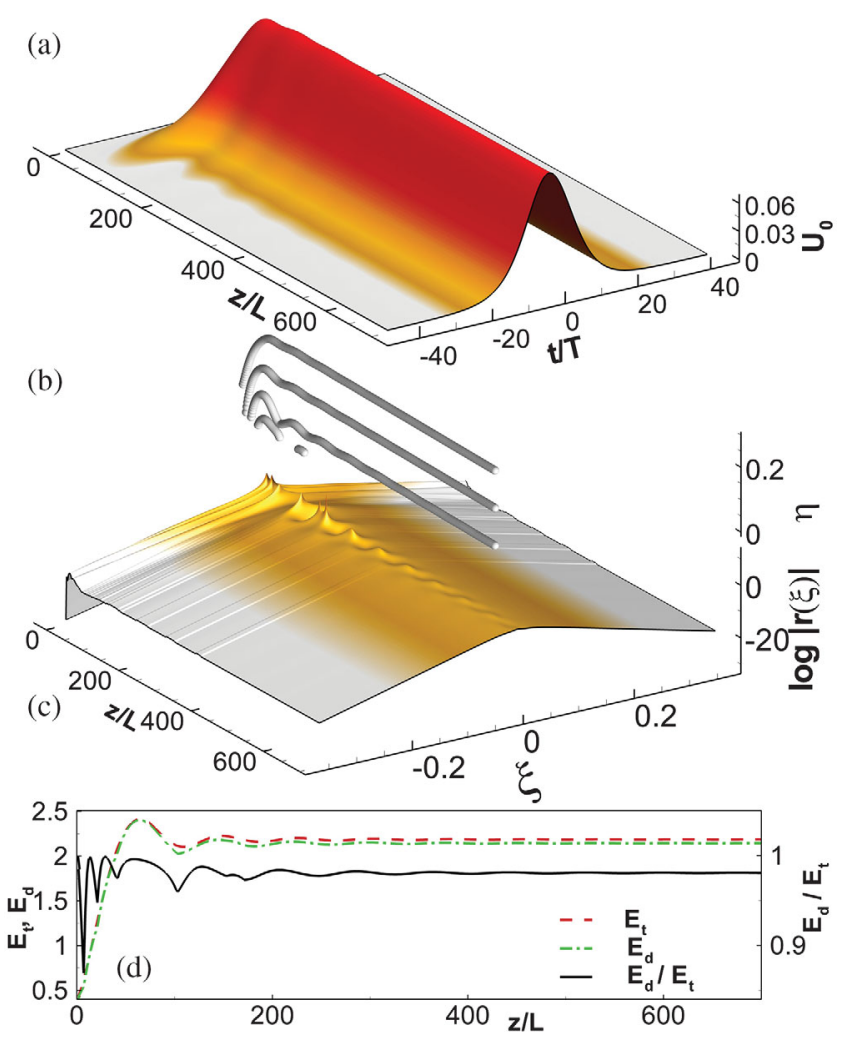

FIG. 4. (a) Evolution of the intensity of the initial pulse having form of the NLSE soliton. Here, $\alpha=3.5, \delta=0.35, \sigma<0$. (b) Dynamics of the discrete eigenvalues $\left\{\zeta_{n}\right\}$ of nonlinear spectrum for the considered case and (c) the logarithm of the continuous nonlinear spectrum $r(\xi)$ module. (d) Evolution of the total energy $E_{t}$, the discrete spectrum energy $E_{d}$, and the ratio $E_{d} / E_{t}$.

nonlinear systems. It is shown that the NFT approach might act as a tool that makes it possible to reduce the number of the effective degrees of freedom when the dynamic is dominated by the coherent structures, even if they are embedded in the dispersive waves and demonstrate unstable behavior. We have presented NFT analysis of chirped solitons, both with arbitrary combination of three parameters (amplitude, chirp, and width), and specific solutions of the GL equation - the so-called autosolitonswith parameters fixed by the coefficients of the GLE. Considering the evolution of the fundamental soliton of the NLSE in the GLE, we have demonstrated that dynamics can be described with high accuracy by a limited number of discrete eigenvalues of the corresponding ZS problem. Our results are in line with the recent experimental works using NFT for characterization of laser radiation [16-18]. One of the most straightforward applications of the NFT analysis is that the nonlinear spectrum defines evolution of any complex input pulse (for example, laser radiation) launched into optical fibers modeled by the NLSE.

This work was supported by the Russian Science Foundation (Grant No. 17-72-30006). 
[1] C. S. Gardner, J. M. Greene, M. D. Kruskal, and R. M. Miura, Phys. Rev. Lett. 19, 1095 (1967).

[2] V. E. Zakharov and A. B. Shabat, J. Exp. Theor. Phys. 34, 62 (1972).

[3] M. J. Ablowitz, D. J. Kaup, A. C. Newell, and H. Segur, Stud. Appl. Math. 53, 249 (1974).

[4] G. L. Lamb, Jr., Elements of Soliton Theory (John Wiley \& Sons, New York, 1980).

[5] M. J. Ablowitz and H. Segur, Solitons and the Inverse Scattering Transform (Society for Industrial and Applied Mathematics, Philadelphia, 1981), p. 425.

[6] S. K. Turitsyn, J. E. Prilepsky, S. T. Le, S. Wahls, L. L. Frumin, M. Kamalian, and S. A. Derevyanko, Optica 4, 307 (2017).

[7] H. Haus, IEEE J. Quantum Electron. 11, 736 (1975).

[8] J. E. Prilepsky, S. A. Derevyanko, and S. K. Turitsyn, J. Opt. Soc. Am. B 24, 1254 (2007).

[9] G. Boffetta and A. Osborne, J. Comput. Phys. 102, 252 (1992).

[10] A. Vasylchenkova, J. E. Prilepsky, and S. K. Turitsyn, Opt. Lett. 43, 3690 (2018).
[11] J. Satsuma and N. Yajima, Prog. Theor. Phys. Suppl. 55, 284 (1974).

[12] F. A. Grunbaum, Inverse Probl. 5, 287 (1989).

[13] A. Tovbis and S. Venakides, Physica (Amsterdam) 146D, 150 (2000).

[14] M. Desaix, L. Helczynski, D. Anderson, and M. Lisak, Phys. Rev. E 65, 056602 (2002).

[15] A. Bambini and P. R. Berman, Phys. Rev. A 23, 2496 (1981).

[16] S. Sugavanam, M. Kamalian, J. Peng, J. E. Prilepsky, and S. K. Turitsyn, in 2017 Conference on Lasers and ElectroOptics Europe \& European Quantum Electronics Conference (CLEO/Europe-EQEC) (IEEE, New York, 2017), Vol. 472, pp. 1-1, https://ieeexplore.ieee.org/ document/8087529.

[17] S. Randoux, P. Suret, A. Chabchoub, B. Kibler, and G. El, Phys. Rev. E 98, 022219 (2018).

[18] P. Ryczkowski, M. Närhi, C. Billet, J.-M. Merolla, G. Genty, and J. M. Dudley, Nat. Photonics 12, 221 (2018). 